\title{
Prevalence of low-energy reporting in the 1930 s
}

\author{
S. Whybrow and J.I. Macdiarmid \\ Rowett Institute, University of Aberdeen, Aberdeen AB25 2ZD
}

Misreporting of foods by study participants when they self-record their diets is a ubiquitous feature of current dietary assessments. Some evidence suggests that the prevalence of misreporting, resulting in apparently low energy intakes (low energy reporting) has increased over the past three decades. Two studies conducted in England during the $1930 \mathrm{~s}^{(1,2)}$ are among the earliest dietary assessments of free-living subjects that used 7-d weighed intakes and rigorous methodology. This study aimed to estimate the prevalence of misreporting in the published data of these two studies.

Subjects (63 men and 63 women) were predominantly students, housewives and professionals in sedentary occupations. Body weight, height and BMI were calculated from the published data ${ }^{(1,2)}$ and BMR was estimated using standard formulae. Probable lowenergy reporters were identified where energy intake:BMR was less than the cut-off value for plausible intakes calculated using occupation relevant physical activity levels ${ }^{(3)}$.

Mean body weight $(65.9$ SD $6.9 \mathrm{~kg})$ and BMI $\left(23.6 \mathrm{SD} 4.3 \mathrm{~kg} / \mathrm{m}^{2}\right)$ were less than typically reported in modern studies and only $25 \%$ had a BMI $>25 \mathrm{~kg} / \mathrm{m}^{2}$. Mean reported daily energy intake (11.0 SD 2.3MJ) and energy intake:BMR (1.71 SD 0.36) were higher than typical modern values in the literature. Only two men and one woman were identified as probable low-energy reporters, equal to $2.3 \%$ of the sample population. This is considerably lower than the prevalence of around $35 \%$, seen in more recent studies. Assuming activity levels were the same in the 1930s as now, as used here, is an obvious limitation. However, reanalysing the data using contemporary estimates of daily energy requirement ${ }^{(4)}$ raised the sample's estimated activity level from $1.64 * \mathrm{BMR}$ to $1.90 * \mathrm{BMR}$, and the prevalence of low-energy reporters to $18.3 \%$.

Potential reasons for an increase in low-energy reporting include a higher prevalence of obesity now. Low energy reporting is often associated with increasing BMI because of conscious dietary restriction, social-desirability, and over-estimation of BMR at higher body weights. Changing meal patterns may have made it easier to forget, or deliberately omit, some foods (e.g. snacks). Low-energy reporting appears to have been far less prevalent in these two early dietary studies than might be expected from more recent experience.

1. Widdowson E (1936) Journal of Hygiene 3, 269-290.

2. Widdowson E \& McCance R (1936) Journal of Hygiene 3, 293-309.

3. Goldberg G, Black A, Jebb S et al. (1991) Eur J Clin Nutr 45, 569-581.

4. Orr J \& Leitch I (1938) Nutrition Abstr \& Rev 7, 509-529. 